\title{
Diaphragm and Lung Ultrasound Indices in Prediction of Outcome of Weaning from Mechanical Ventilation in Pediatric Intensive Care Unit
}

\author{
Dalia A. Abdel Rahman ${ }^{1}$ - S. Saber ${ }^{2}$ - A. El-Maghraby ${ }^{2}$ \\ Received: 4 June 2019 / Accepted: 27 December 2019 / Published online: 8 February 2020 \\ (C) Dr. K C Chaudhuri Foundation 2020
}

\begin{abstract}
Objective To predict the added value of diaphragmatic and lung ultrasound indices (US) in anticipation of the outcome of mechanical ventilation of pediatric patients in the intensive care unit.

Methods This prospective study was conducted in Zagazig University, Pediatric Hospitals, PICU; Egypt. One hundred six mechanically ventilated children aged between 1 mo to 170 mo were included in the study. All patients were candidates for weaning and have been given a chance for spontaneous breathing trial (SBT), during which diaphragmatic and lung US was performed for them. The diaphragm thickening fraction (DTF), the diaphragmatic excursion and the lung US score (LUS), that included 4 US aeration forms, were assessed.

Results There were statistically significant differences between patients with failed weaning and those with successful weaning regarding DTF, diaphragmatic excursion and LUS $(p<0.001)$. The best cut-off value of DTF, diaphragmatic excursion, and LUS for predicting weaning failure was $\geq 23.175 \%, \geq 6.2 \mathrm{~mm}$, and $\geq 12$ with an AUC (area under curve) of $0.932,0.876$, and 0.934 , respectively.

Conclusions Diaphragmatic and lung US add a quick, accurate and non-invasive indices to the weaning readiness parameters compared to the other standard parameters alone. So, it is recommended to be added to the predictive parameters of weaning outcome.
\end{abstract}

Keywords Diaphragmatic and lung ultrasound $\cdot$ Mechanical ventilation $\cdot$ Pediatric intensive care unit $\cdot$ Weaning failure

\section{Introduction}

Weaning from mechanical ventilation (MV) is a challenging decision, it means complete transition from mechanical breathing support to patient's own respiratory drive support. This transition is very critical and needs accurate, intense, confident decision based on trusted validated clinical, radiological and laboratory parameters to avoid the risk of weaning failure [1].

The patient's failure to accomplish this or to withstand $48 \mathrm{~h}$ of spontaneous breathing is defined as a weaning failure. Weaning failure is a disappointing event that, in relation to

Dalia A. Abdel Rahman

daabdelrahman@zu.edu.eg

1 Department of Pediatrics, Faculty of Medicine, Sednawy Hospital, Zagazig University, Zagazig, Egypt

2 Department of Radiology, Faculty of Medicine, Sednawy Hospital, Zagazig University, Zagazig, Egypt the comorbidities and other reported complications, impacts all ICU staff and patient families and costs patient exposure to reintubation risk [2].

The readiness of the patient for weaning depends globally on the respiratory muscles' power that faces the burden of breathing. Unbalance between the power and the burden leads to weaning failure, including any factor that impairs the respiratory muscle function as ICU acquired neuromuscular dysfunction which is either due to prolonged mechanical ventilation, prolonged sedation or any other potential cause [3].

Although the validated respiratory parameters which are assessed during weaning process are appropriate for weaning, however, the proficiency of these parameters to detect the power of the respiratory muscles to carry on the burden of breathing may be deficient [4].

The diaphragm is the principal muscle in the process of respiration. MV for long durations may lead to its atrophy and subsequent impairment of its functions. This atrophy and subsequent dysfunction are the main causes of difficult weaning and subsequently weaning failure [5]. 
The role of Ultrasound (US) in assessment of diaphragmatic function has been conducted in many studies [6], where it can detect the normal and abnormal movements of diaphragm in many different conditions [7]. Also, lung US can be used in the evaluation of the lung condition which is beneficial during making the decision of weaning as it gives us an idea about its aeration and so we can expect if respiratory distress will occur after weaning or not [8].

Few studies implement this tool in assessment of readiness of weaning in pediatrics. In the present study, authors aimed to predict the value of diaphragmatic and lung US indices as new parameters in prediction of the weaning outcome in pediatric age group.

\section{Material and Methods}

This study was approved by the institutional review board of Zagazig University. It was carried out according to the ethical principles of the Declaration of Helsinki.

All parents or guardians of the patients were apprised about the study, and they provided written informed consent.

This study involved 106 children; their age ranged from 1 mo to $170 \mathrm{mo}$, divided according to this age range into: infants, children and adolescents. They were admitted to PICU and connected to mechanical ventilation. During a period from October 2017 through June 2018, all of them were candidates for weaning and following a weaning plan.

The inclusion criteria for this study was: all patients in PICU $<18$ y connected to mechanical ventilation and eligible for weaning in accordance with the following weaning criteria: Signs of reversal of the principle cause of mechanical ventilation; Accepted blood oxygenation i.e., partial pressure of arterial oxygen $\left(\mathrm{PaO}_{2}\right)$ was equal to or more than $60 \mathrm{mmHg}$, partial pressure of arterial carbon dioxide $\left(\mathrm{PaCO}_{2}\right)$ was equal to or less than $45 \mathrm{mmHg}$, fraction of inspired oxygen $\left(\mathrm{FiO}_{2}\right)$ was equal or less than 0.40 , positive end-expiratory pressure (PEEP) was equal to or less than $5 \mathrm{cmH}_{2} \mathrm{O}$ and the ratio of partial pressure of arterial oxygen to the fraction of inspired oxygen was equal or more than 200; Potential of hydrogen $(\mathrm{PH}) \geq 7.30$; $\mathrm{RR} \leq 45 / \mathrm{m}$; Heart rate $(\mathrm{HR}) \leq 140 / \mathrm{min}$; Rapid shallow breathing index $(\mathrm{RSBI}) \leq 8$ breaths $/ \mathrm{min} / \mathrm{ml} / \mathrm{kg}$ body weight; Minimal use of inotropic or vasopressor drugs; Stable body hemodynamics; Adequate consciousness level; Does not receive sedatives or neuromuscular blocking drugs; No fever; Absence of hemorrhage or anemia which means that hemoglobin level was $\geq 7.5 \mathrm{~g} / \mathrm{dl}$, no electrolyte disturbance and specially $\mathrm{K}$ level were above $4 \mathrm{mEq} / \mathrm{dl}[9,10]$.

Exclusion criteria was: Patients having chronic neuromuscular disorder; Patients with known congenital lung or pleural malformation; Patients with unilateral/bilateral absence of diaphragmatic mobility in US; Patients who had undergone diaphragmatic manipulation during thoracic or esophageal surgeries; Major cardiac or abdominal surgery.

All selected patients were submitted to spontaneous breathing trial (SBT). This was done by low level of pressure support ( $5 \mathrm{~cm} \mathrm{H}_{2} \mathrm{O}$ ). The patients were monitored closely for a period 30 to $120 \mathrm{~min}$ to assess the eligibility for weaning [9]. Patients were considered not tolerating the SBT if [10]: RR $>45 / \mathrm{min}$ or change in RR $>50 \%$ above baseline; Arterial oxygen saturation $<90 \%$, arterial oxygen tension $<50 \mathrm{mmHg}$, or increase in arterial carbon dioxide tension $>10 \mathrm{mmHg}$; Increase or decrease in heart rate $>20 \%$ from the baseline; Agitation and diaphoresis; Developing manifestations of increased work of breathing.

If the patient developed any of these manifestations, SBT was ended and the patients were returned to the former settings of MV. Patients were classified into two groups in accordance with their outcome: (1) Weaning failure group: Weaning failure is stated when there is failure of extubation and need of the patient for re-intubation within 24-72 h [11, 12]. (2) Successful weaning group: Patients were successfully extubated with no need for NIV (non-invasive ventilation) or reintubation.

Diaphragmatic US was done during the SBT and score was calculated. It was performed using Logic E9 (GE) and Honda electronics HS-2100 portable US device. The 3.5 MHz convex probe and the 9-11 MHz linear probe were used for patients' examination. The patient lied in semi recumbent position. The linear US probe was placed in the 8th or 9th intercostal space between the anterior and midaxillary line in a perpendicular position to the chest wall. The diaphragm is seen as a threelayered structure (two parallel echogenic layers which represent the pleura and the peritoneum with central hypo-echoic space which stands for the diaphragmatic muscle).

Thickness of the diaphragmatic muscle was measured from the central point of the pleural line to the central point of the peritoneal line in the above-mentioned location during the termination of inspiration and the termination of expiration. This was repeated to take the average and then the diaphragm thickening fraction (DTF) was calculated by the following equation: DTF $=$ (Thickness at the end inspiration - thickness at the end expiration/Thickness at the end expiration $100 \times .($ TI-TE/TE $\times 100)$ [13] (Fig. 1a).

To measure the range of the diaphragmatic movement (diaphragmatic excursion), the convex probe was placed in the subcostal region parallel to the intercostal space by using the M-mode with the cursor crossing the diaphragm and then the highest and lowest peak points were assessed as a marker for the range of diaphragmatic movement (Fig. 1b).

Lung US was performed using a $2.5-5 \mathrm{MHz}$ probe. The upper and lower regions of anterior, posterior and lateral walls of the right and left lungs were examined through the intercostal spaces i.e., 12 regions in total. The worst abnormality detected during US was considered as a characterizing lesion for the examined region. Four US aeration patterns were identified: (1) N, Normal aeration: The presence of lung sliding 
a

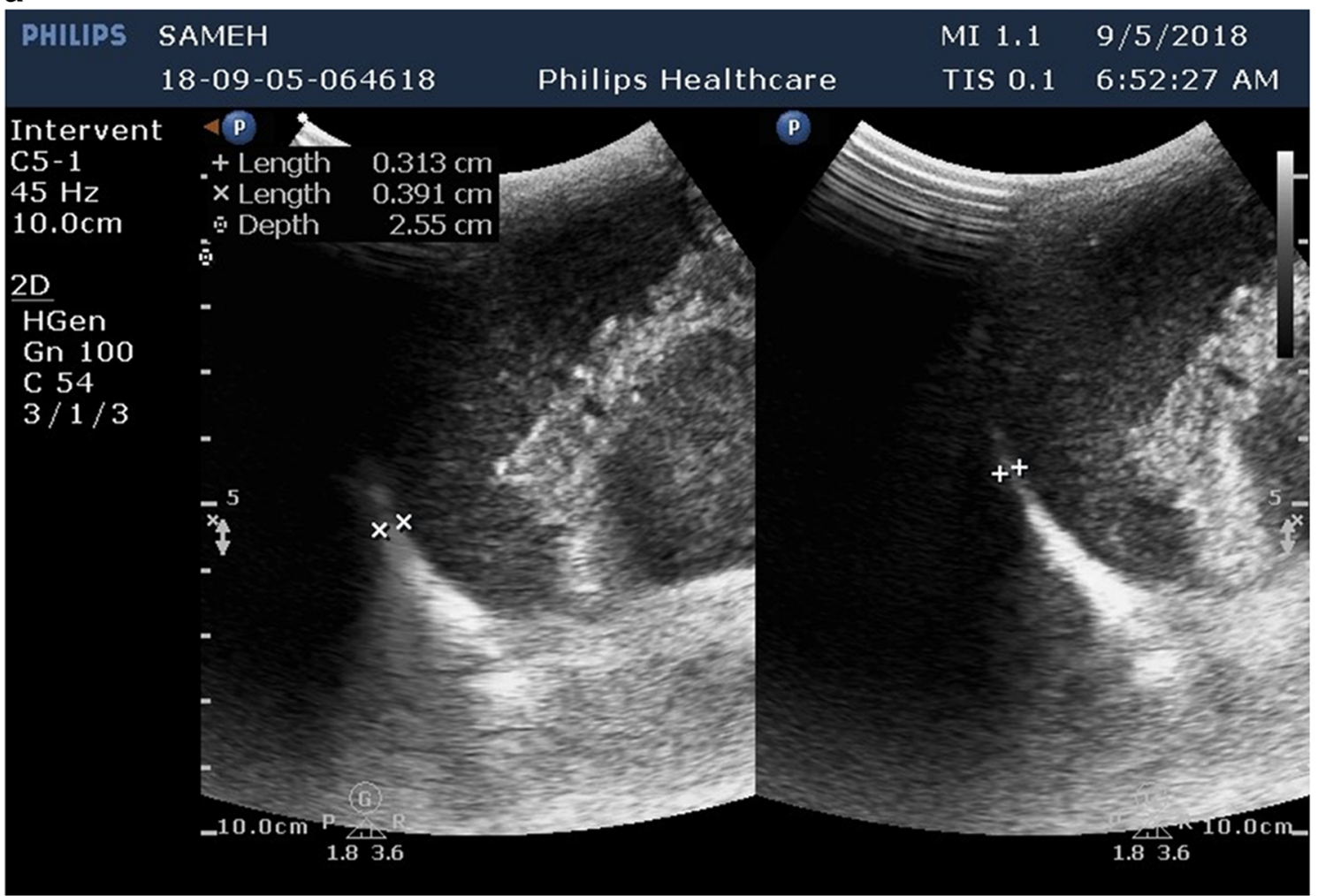

b

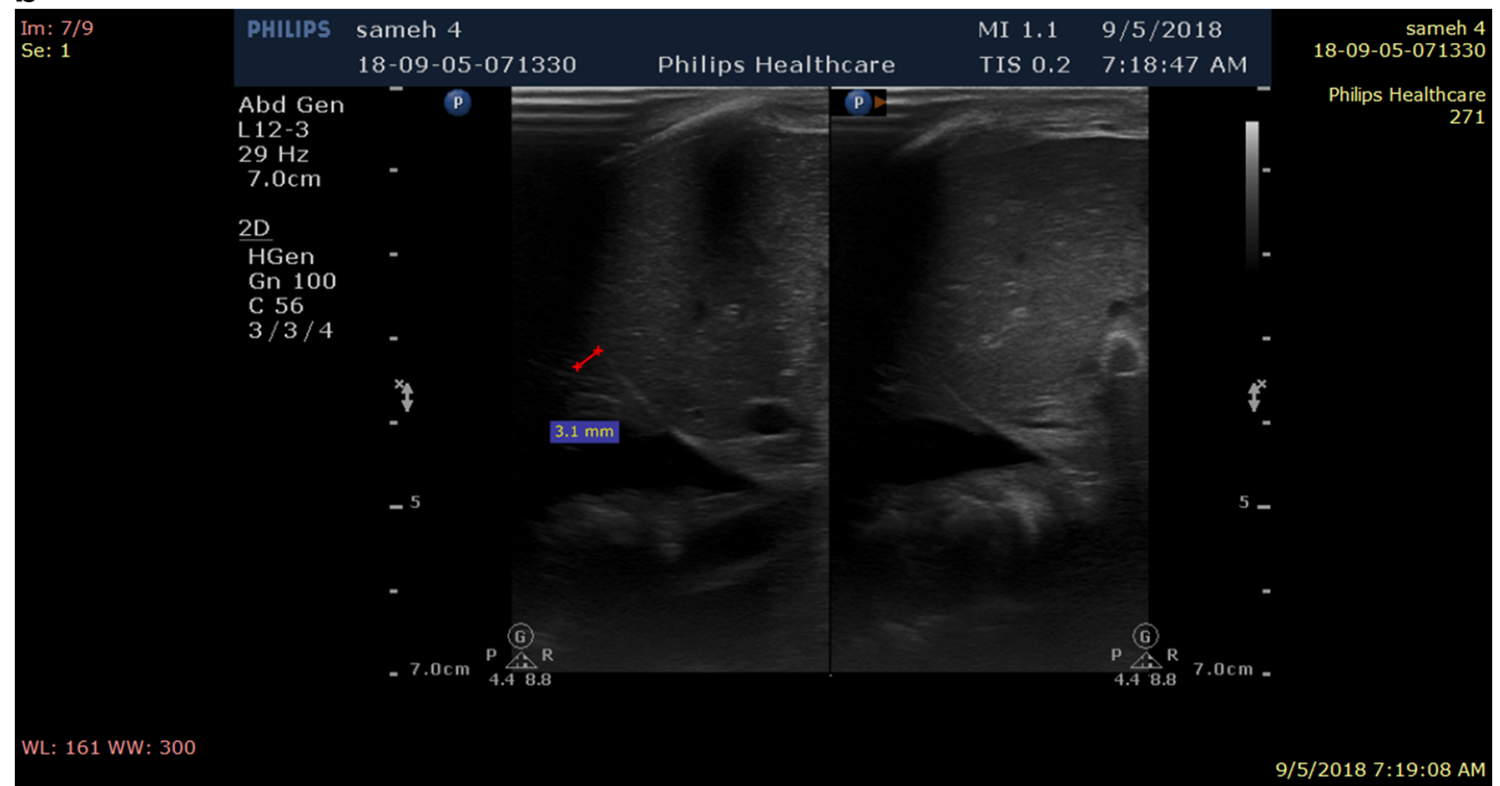

Fig. 1 Ultrasound showing (a) Diaphragmatic thickness measured from the middle of the pleural line to the middle of the peritoneal line at the end of inspiration and at the end of expiration. (b) Diaphragmatic excursion (Distance moved during respiratory cycle)

with A-lines or less than two isolated B lines; (2) B1, moderate loss of lung aeration: Multiple well-defined B lines; (3) B2, severe loss of lung aeration: Multiple abutting B lines; (4) C, lung consolidation: The presence of a tissue pattern characterized by dynamic air bronchogram. A lung US score (LUS) was calculated as follows: $N=0, \mathrm{~B} 1=1, \mathrm{~B} 2=2, \mathrm{C}=3$. This score was calculated ranging from 0 to 36 point for each patient during SBT (Fig. 2) [14].

The collected data were analyzed using SPSS 18.0 for Windows (SPSS Inc., Chicago, IL, USA) and MedCalc 13 for windows (MedCalc Software bvba, Ostend, Belgium). 
Fig. 2 Ultrasound showing calculation of Lung score. $\mathrm{N}$ : Normal lung, B1: Lung staging multiple B lines, B2: Lung staging multiple coalescent $\mathrm{B}$ lines, C: Consolidation
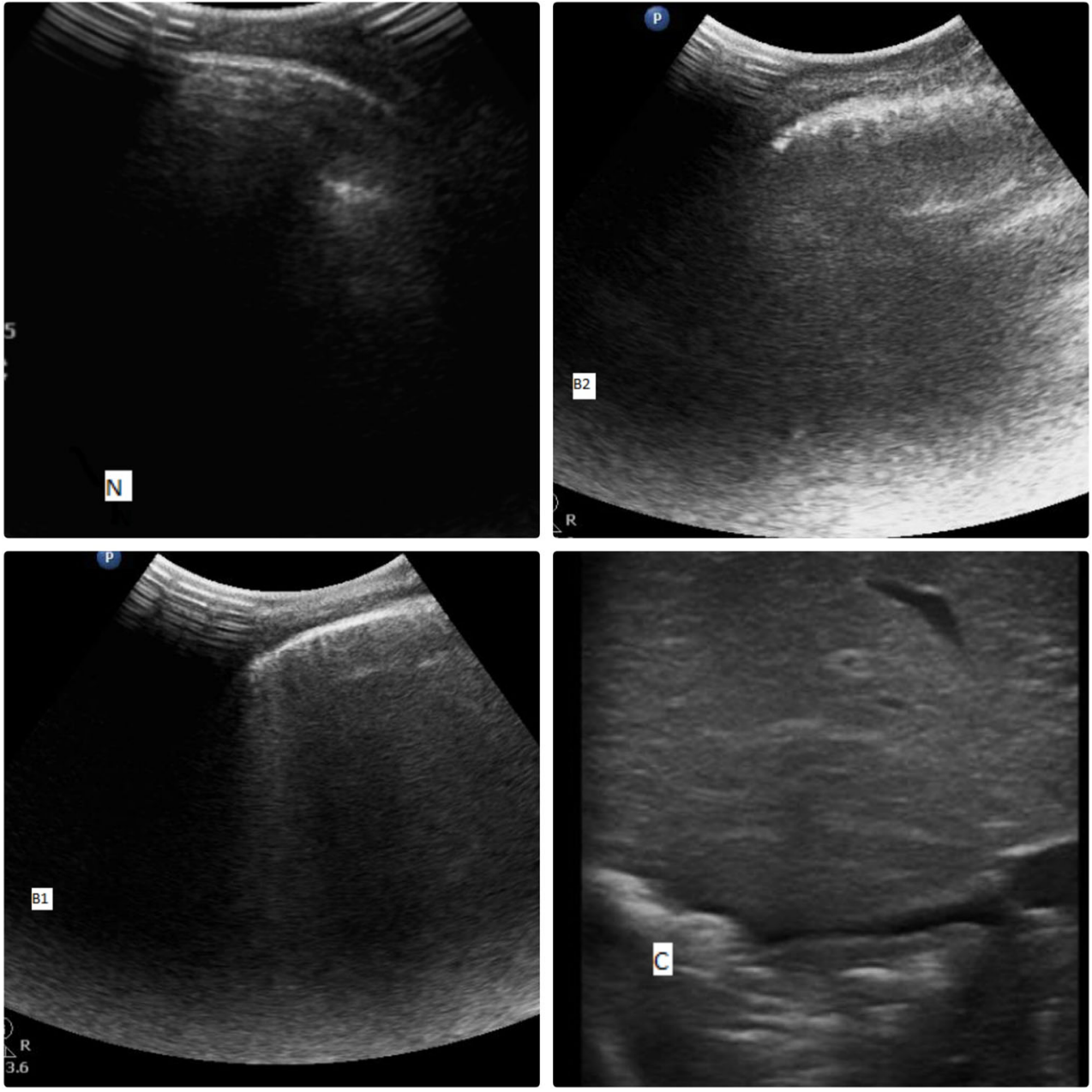

\section{Results}

In this study, 106 children on invasive MV and eligible for SBT were selected. Sixty-four patients (60.4\%) were successfully weaned from MV. Demographic and admission data of studied groups revealed that there were statistically significant differences between patients with failed weaning and those with successful weaning regarding their age, morbidity, days of MV and duration of weaning from MV $(p<0.001)$. Younger patients were more likely for weaning failure. Also, patients with prolonged mechanical ventilation showed more liability for weaning failure. Patients complicated with ventilator associated pneumonia (VAP) or pneumothorax were enviously influenced regarding their weaning outcome. This was detected during the SBT by lung US and obstacled their weaning process and prolonged it (Table 1).

Table 2 shows a comparison between the two studied groups regarding the results of ultrasonography during SBT in infants' age group. There were statistically significant differences between both groups regarding DTF, lung score, and excursion $(p<0.001)$.

Table 3 represents the sonographic data in children age group. DTF is the only parameter which showed significant difference regarding the outcome between the two groups.

In Table 4, no significant difference between the two groups in adolescent age group regarding their sonographic findings is presented.

ROC analysis of the performance of ultrasound parameters for the prediction of weaning failure or success were done (Table 5). The best cut-off value of DTF for predicting weaning success was $\geq 23.175$ with an AUC of 0.932, the application of this threshold resulted in a sensitivity of $100 \%$ and a specificity of $76.2 \%(p<0.001)$. The best cut-off value of excursion for predicting weaning success was $\geq 6.2$ with an AUC of 0.876, the application of this threshold resulted in a sensitivity of $87.5 \%$ and a specificity of $66.7 \%(p<0.001)$. The best cut-off value of LUS for predicting weaning failure was $\geq 12$ with an AUC of 0.934 , the application of this threshold resulted in a sensitivity of $85.7 \%$ and a specificity of $81.2 \%(p<0.001)$. 
Table 1 Demographic and admission data of studied groups

\begin{tabular}{|c|c|c|c|c|}
\hline Characteristics & $\begin{array}{l}\text { Total subjects } \\
n=106(\%)\end{array}$ & $\begin{array}{l}\text { Failed weaning } \\
\text { group } \\
n=42(\%)\end{array}$ & $\begin{array}{l}\text { Successful weaning } \\
\text { group } \\
n=64(\%)\end{array}$ & $p$ value \\
\hline \multicolumn{5}{|l|}{ Age groups } \\
\hline Infants & $84(79.2)$ & $40(47.6)$ & $44(52.4)$ & \multirow[t]{3}{*}{$<0.004^{*}$} \\
\hline Children & $10(9.4)$ & $1(10)$ & $9(90)$ & \\
\hline Adolescents & $12(11.4)$ & $1(8.3)$ & $11(91.7)$ & \\
\hline \multicolumn{5}{|l|}{ Gender } \\
\hline Male & $54(50.9)$ & $20(47.6)$ & $34(53.1)$ & \multirow[t]{2}{*}{0.695} \\
\hline Female & $52(49.1)$ & $22(52.4)$ & $30(46.9)$ & \\
\hline \multicolumn{5}{|l|}{ Cause of admission } \\
\hline Chest problems & $66(62.3)$ & $22(52.4)$ & $44(68.8)$ & \multirow[t]{6}{*}{0.383} \\
\hline Cardiac diseases & $6(5.7)$ & $2(4.8)$ & $4(6.3)$ & \\
\hline Neurological diseases & $8(7.5)$ & $2(4.8)$ & $6(9.4)$ & \\
\hline GIT diseases & $10(9.4)$ & $6(14.3)$ & $4(6.3)$ & \\
\hline Renal complication & $10(9.4)$ & $8(19)$ & $2(3.1)$ & \\
\hline Hematology diseases & $6(5.7)$ & $2(4.8)$ & $4(6.3)$ & \\
\hline \multicolumn{5}{|l|}{ Cause of ventilation } \\
\hline Type I respiratory failure & $28(26.42)$ & $10(23.8)$ & $18(28.1)$ & \multirow[t]{4}{*}{0.599} \\
\hline Type II respiratory failure & $60(56.60)$ & $28(66.7)$ & $32(50)$ & \\
\hline Type III respiratory failure & $8(7.55)$ & $2(4.8)$ & $6(9.4)$ & \\
\hline Type IV respiratory failure & $10(9.43)$ & $2(4.8)$ & $8(12.5)$ & \\
\hline \multicolumn{5}{|l|}{ Exposure to complications } \\
\hline Absent & $82(77.4)$ & $20(47.6)$ & $62(96.9)$ & \multirow[t]{4}{*}{$<0.001^{*}$} \\
\hline VAP & $14(13.2)$ & $14(33.4)$ & $0(0)$ & \\
\hline Pneumothorax & $4(3.8)$ & $4(9.5)$ & $0(0)$ & \\
\hline Post extubation stridor & $6(5.7)$ & $4(9.5)$ & $2(3.1)$ & \\
\hline \multicolumn{5}{|l|}{ Days of MV } \\
\hline Mean \pm SD & $9.25 \pm 4.2$ & $12.81 \pm 7.54$ & $7.44 \pm 3.5$ & \multirow[t]{2}{*}{$<0.001^{*}$} \\
\hline Median (range) & $11(2-25)$ & $12(3-25)$ & $7(2-22)$ & \\
\hline \multicolumn{5}{|l|}{ Duration of weaning (hours) } \\
\hline Mean \pm SD & $7.8 \pm 3.55$ & $9.9 \pm 5.87$ & $7.44 \pm 3.5$ & \multirow[t]{2}{*}{$<0.001^{*}$} \\
\hline Median (range) & $8(1-20)$ & $10(2-20)$ & $7(2-22)$ & \\
\hline
\end{tabular}

GIT Gastrointestinal tract, $M V$ Mechanical ventilation, $S D$ Standard deviation, VAP Ventilator associated pneumonia

* Statistical significance

\section{Discussion}

The validated medical and laboratory parameters for assessment of readiness of patients for weaning from MV are simply applied, but their specificity and sensitivity are limited [15].

It is usual practice now-a-days to do US in ICU or in the emergency department. In this study, authors wondered if the addition of diaphragmatic and lung US improves the performance for prediction of weaning outcome from invasive MV in PICU patients. To reach this goal, 106 children of different age groups admitted to PICU were prospectively examined, ventilated through tracheal tube or tracheostomy and they were ready for weaning according to SBT. By applying diaphragmatic US to those patients during SBT, authors found that during infancy period the measured DTF, diaphragmatic excursion and the total lung score showed highly significant correlations with weaning outcome $(p<0.001)$. These results were matched with results of several studies [16-19].

While in children age group the measured DTF only sonographic parameter showed significant difference between the two studied groups regarding their outcome.

In contrast to the studied adolescent patients, no significant difference was found in the measured ultrasonographic parameters regarding the weaning outcome between the two groups.

In conjunction with present results, Kim et al. [17] concluded that a large number of patients admitted to ICU and 
Table 2 Comparison between the two groups according to the sonographic data detected in infants' age group

\begin{tabular}{lccc}
\hline Parameters & $\begin{array}{l}\text { Failed weaning group } \\
(n=40) \\
{[\text { Mean } \pm \mathrm{SD}]}\end{array}$ & $\begin{array}{l}\text { Successful weaning group } \\
(n=44) \\
{[\text { Mean } \pm \mathrm{SD}]}\end{array}$ & $p$ value \\
\hline Lung score & $14.5 \pm 5.06$ & $9.77 \pm 2.36$ & $<0.001^{*}$ \\
R-Excursion $(\mathrm{mm})$ & $6.06 \pm 1.87$ & $8.21 \pm 2.25$ & $<0.001^{*}$ \\
L-Excursion $(\mathrm{mm})$ & $5.62 \pm 1.55$ & $7.81 \pm 2.1$ & $<0.001^{*}$ \\
Excursion $(\mathrm{mm})$ & $5.84 \pm 1.37$ & $8.01 \pm 1.8$ & $<0.001^{*}$ \\
R-inspiration $(\mathrm{mm})$ & $1.98 \pm 0.82$ & $2.32 \pm 0.62$ & $0.035^{*}$ \\
R-expiration $(\mathrm{mm})$ & $1.68 \pm 0.74$ & $1.75 \pm 0.52$ & 0.610 \\
R-DTF \% & $20.06 \pm 10.41$ & $33.56 \pm 7.81$ & $<0.001^{*}$ \\
L-inspiration $(\mathrm{mm})$ & $1.98 \pm 0.82$ & $2.32 \pm 0.39$ & $0.016^{*}$ \\
L-expiration $(\mathrm{mm})$ & $1.61 \pm 0.51$ & $1.68 \pm 0.28$ & 0.412 \\
L-DTF \% & $20.76 \pm 7.86$ & $29.93 \pm 6.77$ & $<0.001^{*}$ \\
DTF \% & $20.4 \pm 6.24$ & $31.75 \pm 2.93$ & $<0.001^{*}$ \\
\hline
\end{tabular}

DTF Diaphragm thickening fraction, $L$ Left, $R$ Right

* Statistical significance ventilated suffer from new onset of diaphragmatic dysfunction, which is previously normal and which results from long duration of MV and young age of patients. In those patients, failure of weaning is frequent.

The diaphragmatic US may be an effective method in anticipation to know if the patient will develop weaning failure or not. All the previous studies confirmed that diaphragmatic excursion and DTF are helpful indicators for weaning outcome prediction. However, The DTF has a higher sensitivity and better AUC score than excursion so it is more reliable than excursion. This finding is consistent with Umbrello et al. [6] and Baess et al. [14].

Table 3 Relation between sonographic data of studied children age group and their outcome

\begin{tabular}{llll}
\hline Parameters & $\begin{array}{l}\text { Failed weaning } \\
\text { group } \\
(n=1) \\
{[\text { Mean } \pm \mathrm{SD}]}\end{array}$ & $\begin{array}{l}\text { Successful } \\
\text { weaning group } \\
(n=9) \\
{[\text { Mean } \pm \mathrm{SD}]}\end{array}$ & $p$ value \\
\hline Lung score & 30 & $14.22 \pm 6.06$ & 0.2 \\
R-Excursion $(\mathrm{mm})$ & 4 & $9.22 \pm 2.22$ & 0.064 \\
L-Excursion $(\mathrm{mm})$ & 7 & $8.64 \pm 1.32$ & 0.273 \\
Excursion $(\mathrm{mm})$ & 5.5 & $8.83 \pm 1.71$ & 0.102 \\
R-inspiration $(\mathrm{mm})$ & 2.8 & $2.96 \pm 0.27$ & 0.753 \\
R-expiration $(\mathrm{mm})$ & 2.3 & $2.34 \pm 0.41$ & 0.921 \\
R-DTF \% & 21.74 & $26.49 \pm 3.47$ & 0.23 \\
L-inspiration $(\mathrm{mm})$ & 2.3 & $2.96 \pm 0.27$ & 0.052 \\
L-expiration $(\mathrm{mm})$ & 2.1 & $2.26 \pm 0.13$ & 0.284 \\
L-DTF \% & 9.52 & $30.93 \pm 8.43$ & $0.043^{*}$ \\
DTF \% & 15.63 & $28.71 \pm 5.21$ & $0.044^{*}$ \\
\hline
\end{tabular}

DTF Diaphragm thickening fraction, $L$ Left, $R$ Right

* Statistical significance
In the present study, the diaphragmatic excursion cut-off value was $6.2 \mathrm{~mm}$ with $87.5 \%$ sensitivity and $66.7 \%$ specificity. This agrees with results of other studies which reported that the cut-off value of Diaphragmatic E in the assessment of the weaning outcome is $10-11 \mathrm{~mm}$ with different degrees of sensitivity and specificity. Saeed et al. [16] stated that it has $86.4 \%$ sensitivity and $87.5 \%$ specificity while Baess et al. stated that it has $69.5 \%$ sensitivity and $71.4 \%$ specificity [14].

Regarding the DTF in present study, at a cut-off value of $23.175 \%$, the sensitivity and specificity were $100 \%$ and $76.2 \%$, respectively. This agrees with Umbrello et al. [6] whose results showed a cut-off value of $20 \%$. Also, Baess et al. [14] and Di Nino et al. [18] reported a 30\% DTF cut-

Table 4 Relation between sonographic data of adolescent patients and fate of weaning

\begin{tabular}{llcc}
\hline Parameters & $\begin{array}{l}\text { Failed weaning } \\
\text { group }(\mathrm{n}=1) \\
{[\text { Mean } \pm \mathrm{SD}]}\end{array}$ & $\begin{array}{l}\text { Successful weaning } \\
\text { group }(n=11) \\
{[\text { Mean } \pm \mathrm{SD}]}\end{array}$ & $p$ value \\
\hline Lung score & 11 & $10.09 \pm 2.3$ & 561 \\
R-Excursion (mm) & 14 & $11.6 \pm 1.16$ & 0.076 \\
L-Excursion (mm) & 12.2 & $11.85 \pm 0.99$ & 0.745 \\
Excursion (mm) & 13.1 & $11.73 \pm 0.67$ & 0.077 \\
R-inspiration (mm) & 3.1 & $2.93 \pm 0.19$ & 0.402 \\
R-expiration (mm) & 2.3 & $2.21 \pm 0.13$ & 0.518 \\
R-DTF \% & 34.78 & $32.58 \pm 5.48$ & 0.709 \\
L-inspiration (mm) & 2.9 & $2.88 \pm 0.15$ & 0.908 \\
L-expiration (mm) & 2.4 & $2.2 \pm 0.18$ & 0.324 \\
L-DTF \% & 20.83 & $31.49 \pm 8.53$ & 0.259 \\
DTF \% & 27.81 & $32.04 \pm 4.32$ & 0.371 \\
\hline
\end{tabular}

DTF Diaphragm thickening fraction, $L$ Left, $R$ Right 
Table 5 Performance characteristics of ultrasound parameters in predicting failure of weaning

\begin{tabular}{lcccccccc}
\hline Parameter & Cut-off & AUC & Sensitivity & Specificity & PPV & NPV & Accuracy & $p$ value \\
\hline Total lung score & 12 & 0.934 & 85.7 & 81.2 & 75 & 89.7 & 83.02 & $<0.001^{*}$ \\
DTF (mm) & 23.175 & 0.932 & 100 & 76.2 & 86.5 & 100 & 90.6 & $<0.001^{*}$ \\
R-TF (mm) & 21.981 & 0.872 & 93.8 & 71.4 & 83.3 & 88.2 & 84.9 & $<0.001^{*}$ \\
L-TF (mm) & 19.918 & 0.831 & 96.9 & 61.9 & 79.5 & 92.9 & 83 & $<0.001^{*}$ \\
Excursion (mm) & 6.2 & 0.876 & 87.5 & 66.7 & 77.8 & 76.5 & 77.4 & $<0.001^{*}$ \\
R- Excursion (mm) & 6.5 & 0.827 & 81.2 & 66.7 & 78.7 & 70 & 75.5 & $<0.001^{*}$ \\
L- Excursion (mm) & 6.1 & 0.841 & 81.2 & 57.1 & 74.3 & 66.7 & 71.7 & $<0.001^{*}$ \\
\hline
\end{tabular}

AUC Area under curve, $D T F$ Diaphragm thickening fraction, $L$ Left, $N P V$ Negative predictive value, $P P V$ Positive predictive value, $R$ Right

${ }^{*}$ Statistical significance off value with a sensitivity and specificity of $69.57 \%, 88 \%$, and $71.43 \%$, and $71 \%$, respectively. However, this differs from Ferrari et al. [19] whose results showed higher cut-off value of $36 \%$ with a sensitivity and specificity of $82 \%$ and $88 \%$, respectively.

The current study reveals that LUS shows a promising role as a predictor of SBT outcome and for expecting the postextubation distress. The mean values of this score were significantly higher in the failed group $(18 \pm 4.71)$ than in the success group $(10.16 \pm 2.37)(p<0.001)$. The sensitivity and specificity of this score for predicting weaning failure at a cutoff point of 12 were $85.7 \%$ and $81.2 \%$, respectively. The present results are close to results published by Soummer et al. [9] which showed that LUS can predict weaning outcome with $90 \%$ sensitivity and specificity at a score $>13$.

In the same panel of present results, Soummer et al. [9] studied the role of lung US in detecting lung aeration during post-extubation stress in 100 patients and found that less lung aeration usually means re-intubation i.e., weaning failure. In another research done by Perbet et al. [20], authors evaluated the role of the soluble form of the receptor for advanced glycogen end products (SRAGE) as a serum marker to denote lung epithelial injury and ineffective lung aeration in cases of post-extubation stress. In this study, they depended on lung US as a standard predictor of lung aeration. This highlights the growing evidence and builts trust about the validity of US in the detection of lung aeration and its role in post-extubation outcome.

The encouraging results of present study are highlighting the promising role of bedside ultrasound chest in the prediction of ventilator weaning and this may be one of the steps that build up the era of weaning parameters. The hope of reaching the ideal tool to pinch mark the suitable time to wean the ventilator is not far in PICU practice. Further researches in this area may prove that addition of chest US as a parameter for accurate estimation of weaning readiness parameters is one of the possible solutions for this problem.
So, authors conclude that diaphragmatic and lung US is a quick and non-harmful technique for prediction of weaning success with highly accurate results compared to the other traditional medical parameters alone as we may face many cases ready for weaning by traditional parameters but which failed with weaning process progress. So, they can be added to the conventional parameters for accurate prediction of the weaning outcome.

But this study has some limitations regarding nonhomogeneity of its data, as most of the patients fulfilled the inclusion criteria during the study period were infants, so the data does not accurately represent children and adolescents age group. Also, the sample size is relatively small. So larger studies or multicenter studies including all age groups are recommended for more reliable accurate data to detect the exact range of cut-offs of the ultrasonographic measurements to include them with the validated weaning parameters in PICU protocols.

Acknowledgements Dr. Amany Abdullah, Lecturer of Community Medicine helped in statistical analysis.

Authors' Contribution DAAR: Concept and design of the study, collected, analysed and interpreted the data; SS: Data collection, did the ultrasound, interpretation and analysis of its data; AE-M and DAAR: Drafted the article and revised it critically for important intellectual content. All authors have approved the final version of the manuscript. Professor Dr. Alaa Ziedan, Manager of PICU- Pediatric Department, Zagazig University is the guarantor for this paper.

\section{Compliance with Ethical Standards}

Conflict of Interest None.

\section{References}

1. Khemani RG, Hotz J, Morzov R, et al. Pediatric extubation readiness tests should not use pressure support. Intensive Care Med. 2016;42:1214-22. 
2. Pérez-Calatayud ÁA, Carrillo-Esper R, Arch-Tirado E. Quantitative evaluation proposal of a ultrasonographic protocol for weaning from mechanical ventilation. Gac Med Mex. 2016;152:273-80.

3. Zein H, Baratloo A, Negida A, Safari S. Ventilator weaning and spontaneous breathing trials; an educational review. Emerg (Tehran). 2016;4:65-71.

4. Shoaeir M, Noeam KM, Mahrous A, Alaa A. Lung aeration loss as a predictor of re-intubation using lung ultrasound in mechanically ventilated patients. Biolife. 2016;4:514-20.

5. Robriquet L, Georges H, Leroy O, Devos P, D'escrivan T, Guery B. Predictors of extubation failure in patients with chronic obstructive pulmonary disease. J Crit Care. 2006;21:185-90.

6. Umbrello M, Formenti P, Longhi D, et al. Diaphragm ultrasound as indicator of respiratory effort in critically ill patients undergoing assisted mechanical ventilation: a pilot clinical study. Crit Care. 2015;19:161

7. Mayo P, Volpicelli G, Lerolle N, Schreiber A, Doelken P, VieillardBaron A. Ultrasonography evaluation during the weaning process: the heart, the diaphragm, the pleura and the lung. Intensive Care Med. 2016;42:1107-17.

8. Summerhill EM, El-Sameed YA, Glidden TJ, McCool FD. Monitoring recovery from diaphragm paralysis with ultrasound. Chest. 2008;133:737-43.

9. Soummer A, Perbet S, Brisson H, et al. Ultrasound assessment of lung aeration loss during a successful weaning trial predicts postextubation distress. Crit Care Med. 2012;40:2064-72.

10. Enghard P, Rademacher S, Nee J, et al. Simplified lung ultrasound protocol shows excellent prediction of extravascular lung water in ventilated intensive care patients. Crit Care. 2015;19:36.

11. Teixeira C, Teixeira PJ, de Leon PP, Oliveira ES. Work of breathing during successful spontaneous breathing trial. J Crit Care. 2009;24: 508-14.
12. Saraogi A. Lung ultrasound: present and future. Lung India. 2015;32:250-7.

13. Stawicki SP. Mechanical ventilation: weaning and extubation. Int J Acad Med. 2017;3:67-71.

14. Baess AI, Abdallah TH, Emara DM, Hassan M. Diaphragmatic ultrasound as a predictor of successful extubation from mechanical ventilation: thickness, displacement, or both? Egypt J Bronchol. 2016;10:162-6.

15. Jeong BH, Ko MG, Nam J, et al. Differences in clinical outcomes according to weaning classifications in medical intensive care units. PLoS One. 2015;10:e0122810.

16. Saeed AM, El Assal GI, Ali TM, Hendawy MM. Role of ultrasound in assessment of diaphragmatic function in chronic obstructive pulmonary disease patients during weaning from mechanical ventilation. Egypt J Bronchol. 2016;10:167-72.

17. Kim WY, Suh HJ, Hong SB, Koh Y, Lim CM. Diaphragm dysfunction assessed by ultrasonography: influence on weaning from mechanical ventilation. Crit Care Med. 2011;39:2627-30.

18. DiNino E, Gartman EJ, Sethi JM, McCool FD. Diaphragm ultrasound as a predictor of successful extubation from mechanical ventilation. Thorax. 2014;69:423-7.

19. Ferrari G, De Filippi G, Elia F, Panero F, Volpicelli G, Aprà F. Diaphragm ultrasound as a new index of discontinuation from mechanical ventilation. Crit Ultrasound J. 2014;6:8.

20. Jabaudon M, Perbet S, Soummer A, et al. Plasma levels of SRAGE, do not predict post-extubation distress after a successful weaning trial. Am J Respir Crit Care Med. 2012;185:A3093.

Publisher's Note Springer Nature remains neutral with regard to jurisdictional claims in published maps and institutional affiliations. 